\title{
Inhibition of Ligand-Gated TRPA1 by General Anesthetics
}

\author{
Hoai T. Ton, Thieu X. Phan, and Gerard P. Ahern \\ Department of Pharmacology and Physiology, Georgetown University, Washington DC (H.T.T., T.X.P., G.P.A.) and Department \\ of Biology, Vinh University, Vinh city, Nghe An, Vietnam (H.T.T., T.X.P.)
}

Received November 10, 2019; accepted May 27, 2020

\begin{abstract}
Several general anesthetics (GAs) produce pain or irritation upon administration, and this occurs predominantly through the activation of the nociceptive ion channel, transient receptor potential ankyrin type 1 (TRPA1). However, the effects of GAs on agonist-mediated TRPA1 activity are unclear. Here we show that a diverse range of noxious and non-noxious volatile anesthetics, at clinically relevant concentrations, inhibit ligand-activated TRPA1 currents. These effects are species-specific; GAs blocks rodent TRPA1 without affecting the Drosophila ortholog. Furthermore, propofol inhibits rodent but not human TRPA1. Analysis of chimeric TRPA1 proteins and mutagenesis combined reveals two amino acid residues located in the S5 domain, Ser876 and Thr877, that are critical for the inhibitory effects of isoflurane and propofol. Introduction of these residues into
\end{abstract}

Drosophila TRPA1 confers anesthetic inhibition. Furthermore, several residues lining the presumptive binding pocket for noxious GAs are not required for the inhibitory effects of GAs. We conclude that anesthetics inhibit TRPA1 by interacting at a site distinct from the activation site. The inhibitory effects of GAs at TRPA1 may contribute to the diverse pharmacological action of these drugs.

\section{SIGNIFICANCE STATEMENT}

We show that both noxious and non-noxious general anesthetics inhibit agonist-evoked transient receptor potential ankyrin type 1 (TRPA1) activity and identify critical amino acid residues located in the pore domain. Inhibition of TRPA1 may affect pain and vascular signaling during anesthesia.

\section{Introduction}

General anesthetics (GAs) are an important class of drug that produce reversible unconsciousness by acting on diverse protein targets in the nervous system. Additionally, some GAs are noxious, activating sensory nerves to cause pain upon injection (Tan and Onsiong, 1998; Picard and Tramèr, 2000) or respiratory irritation upon inhalation (TerRiet et al., 2000; von Ungern-Sternberg et al., 2008). The principal sensory nerve target for GAs is the transient receptor potential ankyrin type 1 (TRPA1) ion channel (Matta et al., 2008; Eilers et al., 2010; Fischer et al., 2010). Disruption of the TRPA1 gene abolishes propofol-evoked vascular "pain" (Matta et al., 2008), isoflurane-induced mechanical hyperalgesia (Eilers et al., 2010), and desflurane-induced bronchoconstriction (Satoh and Yamakage, 2009; Eilers et al., 2010; Mutoh et al., 2013). Furthermore, mice lacking TRPA1 exhibit a shorter onset for isoflurane-induced anesthesia associated with less respiratory irritation, compared with wild-type mice or TRPV1-null mice (Li et al., 2015). In addition to expression in sensory nerves, TRPA1 is located in vascular endothelial cells, and activation of vascular TRPA1 and subsequent production of nitric oxide contributes to the vasorelaxant effects of propofol (Sinha et al., 2015). Thus, modulation of

This study was supported by the National Institutes of Health [Grant R21NR012065].

https://doi.org/10.1124/mol.119.118851.
TRPA1 contributes significantly to the spectrum of pharmacological effects of anesthetics.

Recent studies have identified a putative binding site for anesthetics in TRPA1 (Ton et al., 2017; Woll et al., 2017). Ton et al. (2017) showed that propofol and isoflurane share with the antagonist A-967079 a molecular binding pocket located between the transmembrane S5, S6 and pore helix domains. In contrast, Drosophila TRPA1 (dTRPA1) lacks this binding site, but introduction of amino acid residues that line this pocket confers anesthetic sensitivity. Furthermore, molecular modeling revealed that isoflurane and propofol bind to this cavity by forming H-bond, halogen-bond, and sulfur aromatic interactions with Ser876, Met915, and Met956 in human TRPA1. Mutating Met915 and Met956 abrogated anesthetic activation without affecting the effects of menthol or A-967079, indicating these residues are selective targets for anesthetics. Furthermore, Woll et al. (2017) used photoaffinity labeling with meta-aziproprofol to directly interrogate the propofol binding site. This revealed two photolabeled residues, Val954 and Glu969, in the S6 helix of mouse TRPA1 and supports the A-967079 cavity as a positive modulatory site for propofol (Woll et al., 2017).

Notably, although pungent anesthetics activate TRPA1, these drugs also produce a surge in current upon washout suggesting an additional inhibitory effect (Matta et al., 2008; Fischer et al., 2010). Furthermore, single channel recordings determined a reduction in mean channel conductance consistent with a pore-blocking mechanism (Matta et al., 2008). 
Thus, these data suggest that anesthetics possess both agonistic and antagonistic properties. Whether these effects are restricted to noxious anesthetics is unclear. Here we show that both noxious and non-noxious anesthetics inhibit TRPA1-mediated currents at clinically relevant concentrations. Furthermore, we identify critical residues located in the S5 transmembrane domain required for anesthetic-induced inhibition.

\section{Materials and Methods}

Cell Culture. HEK293F cells were cultured in Dulbecco's modified Eagle's medium supplemented with $10 \%$ fetal bovine serum, $1 \mathrm{mM}$ sodium pyruvate, $1 \% 100 \times$ minimal essential medium nonessential amino acids, and 1\% penicillin/streptomycin (HyClone, UT) at $37^{\circ} \mathrm{C}$ in a water-saturated atmosphere containing $5 \% \mathrm{CO}_{2}$. Cell cultures were seeded in a culture flask $\left(25 \mathrm{~cm}^{2}\right)$ (Sarstedt Inc., Nümbrecht, Germany) and subcultured twice a week. For electrophysiology, cells were plated on poly-D-lysine-coated coverslips, transiently transfected with wild-type or mutant TRPA1 and GFP using lipofectamine, and used for experiments within 1 to 2 days. Cells were routinely tested and were negative for mycoplasma.

Site-Directed Mutagenesis and Chimeras. Human, rat, and mouse TRPA1 were gifts from David Julius (University of California, San Francisco, CA). Drosophila TRPA1 was a gift from Paul Garrity (Brandeis University, Waltham, MA). Chimeric Drosophila-mouse TRPA1 constructs were a gift from Ardem Patapoutian (Scripps Research Institute, La Jolla, CA). TRPA1 mutants were generated by polymerase chain reaction mutagenesis using QuikChange SiteDirected Mutagenesis Kit (Agilent Technologies, Santa Clara, CA), and the sequences were confirmed by sequencing.

Electrophysiology. Whole-cell, voltage-clamp recordings were performed by using an EPC8 patch-clamp amplifier (HEKA Electronics, Lambrecht, Germany) that was controlled by the program Pulse (version 8.65; HEKA Electronics). The bath solution contained $140 \mathrm{mM} \mathrm{NaCl}, 4 \mathrm{mM} \mathrm{KCl}, 1 \mathrm{mM} \mathrm{MgCl}_{2}, 1.2 \mathrm{mM} \mathrm{CaCl}_{2}, 10 \mathrm{mM}$ HEPES, and $5 \mathrm{mM}$ glucose, $\mathrm{pH}$ 7.3. The pipette solution contained $140 \mathrm{mM} \mathrm{CsCl}, 10 \mathrm{mM}$ HEPES, $10 \mathrm{mM}$ EGTA, and $2 \mathrm{mM} \mathrm{Mg}$-ATP, $\mathrm{pH}$ 7.3. Solutions were applied via a valve-controlled gravity-fed perfusion system. A 200 -millisecond ramp from -100 to $+100 \mathrm{mV}$ was used to evoke the current and measured the voltage-dependent properties of the channels. After the allyl isothiocyanate (AITC)-evoked current stabilized, we measured the current responses to AITC plus anesthetics within 3-5 seconds of application. The anesthetic was then washed out to confirm reversibility of inhibition. The control current was calculated from the average of the amplitude measured immediately before and after the anesthetic application.

Volatile General Anesthetics and Chemicals. Saturated stock solutions of volatile GAs were prepared in gas-tight bottles by dissolving excess anesthetic agents in bath solutions overnight as previously described (Matta et al., 2008). From these stock solutions, fresh dilutions were made up every 40-60 minutes. Concentrations of GAs in the bath solutions were verified by using a modified head-space gas chromatography method. Allyl isothiocyanate was from Sigma (St. Loius, MO), and propofol was from Thermor Fisher Scientific (Waltham, MA).

Statistical Analysis. Data were analyzed using Prism (GraphPad Software, La Jolla, CA) and are presented as means \pm S.D. Statistical significance was assessed with one-way ANOVA, with treatment interactions assessed by Tukey's post hoc multiple comparisons test. The sample sizes were adjusted after initial data collection. The study was exploratory in nature and did not test a prespecified statistical null hypothesis; the $P$ values are therefore descriptive.

\section{Results}

General Anesthetics Inhibit Agonist-Evoked Activation of TRPA1. Previous studies have revealed that noxious
GAs are partial agonists at TRPA1; however, it is unclear whether GAs affect ligand-gated TRPA1 function. We therefore explored the effects of isoflurane on TRPA1 currents activated by the potent electrophilic agonist, AITC. Application of isoflurane alone $(0.9 \mathrm{mM})$ to HEK293 cells expressing rat TRPA1 produced an outwardly rectified current, whereas a saturating concentration of AITC produced a near-ohmic response. Notably, coapplication of isoflurane nearly completely inhibited the AITC-evoked current (Fig. 1, A and B). No responses to AITC were observed in mock-transfected cells demonstrating specificity for TRPA1 (data not shown). Next, we screened a range of GAs for antagonistic effects at TRPA1, including halothane, sevoflurane, diethyl ether, chloroform, propofol, and the long-chain alcohol, octanol. First, we verified whether these compounds exhibited agonistic effects. Figure 1C summarizes the agonist activity (expressed as a percentage of AITC) at both negative and positive holding potential. As expected, only known pungent agents (isoflurane, propofol, and octanol) activated TRPA1. Next, we tested the effects of GAs on AITC-evoked activity. Figure 1D shows that a variety of GAs (with the exception of diethyl ether) inhibited AITC-evoked currents $(P<0.05$ for each). The antagonism was more pronounced at negative (Fig. 1D, upper panel) compared with positive voltages (Fig. 1D, lower panel), consistent with the voltage dependence we have previously documented with pungent GAs (Matta et al., 2008). Thus, these data reveal that although a subset of GAs are partial agonists, a broad spectrum of GAs are capable of inhibiting agonist-evoked activation of TRPA1.

Next, we asked whether this inhibition of TRPA1 occurs at relevant anesthetic concentrations. Figure 2 shows the concentration dependence for isoflurane, halothane, and sevoflurane to inhibit AITC-evoked currents. The smooth lines are the best fits to a Hill equation yielding an $\mathrm{IC}_{50}$ of $0.28,0.27$, and $0.43 \mathrm{mM}$ [ 1 minimum alveolar concentration (MAC)] for isoflurane, halothane, and sevoflurane, respectively. Thus, relevant pharmacological concentrations of inhalational agents are sufficient to inhibit agonist-evoked TRPA1 activity.

Species-Specific Differences in Response to Isoflurane and Propofol. Next, we tested TRPA1 orthologs from rat, human, and Drosophila for sensitivity to isoflurane and propofol. Interestingly, our data revealed that isoflurane and propofol modulated TRPA1 in a species-specific manner. Figure 3 shows that whereas isoflurane $(0.9 \mathrm{mM}, \sim 3 \mathrm{MAC})$ inhibited human and rat TRPA1, dTRPA1 in contrast was completely insensitive to isoflurane (up to $5 \mathrm{mM}$ ). Furthermore, propofol $(100 \mu \mathrm{M})$,selectively inhibited rat TRPA1, with no apparent blockade of the human or Drosophila channel (Fig. 3).

The S5 Domain of TRPA1 Is Critical for the Inhibitory Effects of Isoflurane. Previously we exploited mouse-Drosophila TRPA1 chimeras to identify a critical domain for activation by anesthetics (Ton et al., 2017). Since rodent and Drosophila TRPA1 also exhibit differential inhibitory responses to isoflurane, we reasoned that we could use a similar approach to identify essential domain(s) required for inhibition. Similar to rat TRPA1 we found that isoflurane $(0.9 \mathrm{mM})$ markedly inhibited mouse TRPA1 currents by $92.4 \%$ ( $n=3 ; P<0.01$, one-way ANOVA). We found that exchanging the Drosophila $\mathrm{N}$ terminus with its mouse counterpart (dTRPA1-mN chimera) did not confer sensitivity to isoflurane (no detectable inhibition, $n=4$ ). However, introducing the Drosophila S5 domain into the mouse channel (mTRPA1-dS5 chimera) abolished the inhibitory effect of 


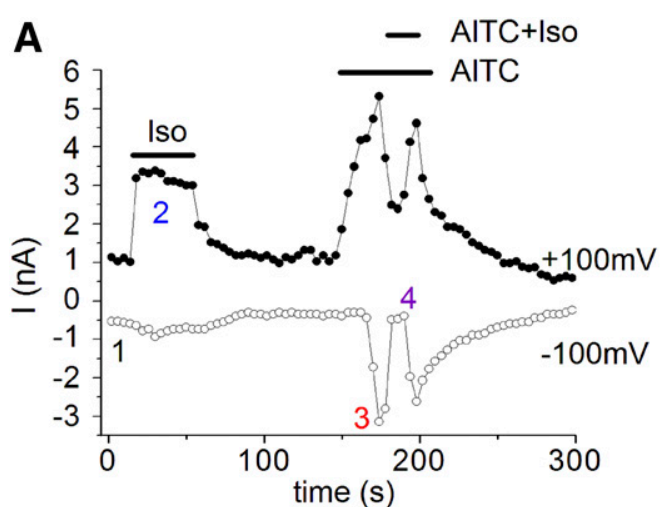

C

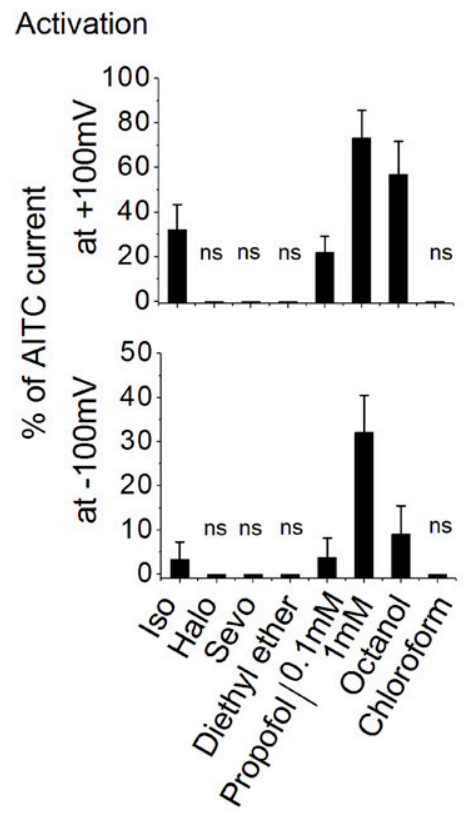

B

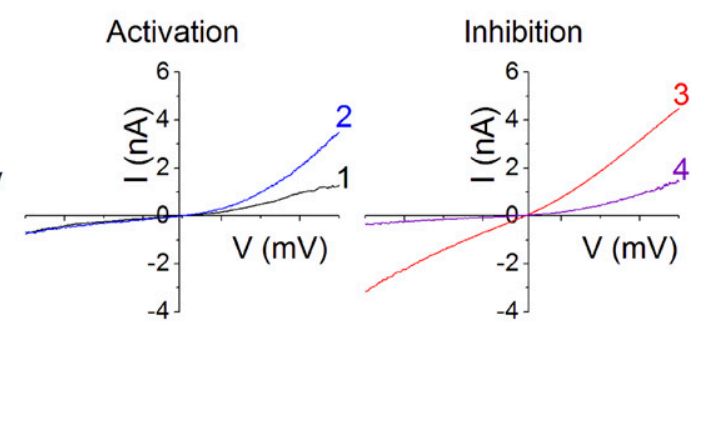

D

Inhibition

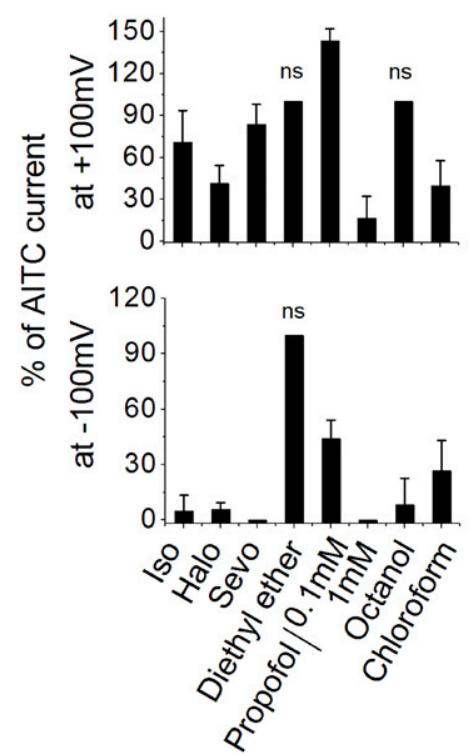

Fig. 1. GAs inhibit agonist-evoked activation of TRPA1. (A) Time-course recording of voltage ramps from -100 to $+100 \mathrm{mV}$ applied every 4 seconds; current measured at -100 (open circles) and $+100 \mathrm{mV}$ (filled circles) during each ramp is plotted in response to isoflurane (Iso; $0.9 \mathrm{mM}$ ) and AITC (100 $\mu \mathrm{M}$ ) indicated by the horizontal bars. (B) Current-voltage (I-V) relationships recorded from the same cell by the voltage ramp collected at basal level (1), the peak isoflurane response (2), the peak AITC response (3), and AITC plus isoflurane response (4) (indicated in A). (C, D) Summary of activation (C) of TRPA1 and block (D) of AITC-evoked currents at +100 and $-100 \mathrm{mV}$ by different GAs: isoflurane (Iso; $0.9 \mathrm{mM} ; n=8)$, halothane (Halo; $0.9 \mathrm{mM} ; n=3$ ), sevoflurane (Sevo; $0.9 \mathrm{mM} ; n=5)$, diethyl ether ( $5 \mathrm{mM} ; n=3)$, propofol (100 $\mu \mathrm{M}$ and $1 \mathrm{mM} ; n=4)$, octanol ( $2 \mathrm{mM} ; n=3)$, and chloroform ( $1 \mathrm{mM} ; n=4)$. The action of GA was normalized to AITC-evoked current in each cell. Except where otherwise indicated (ns, not significant), all differences were statistically significant compared with control $(P<0.05$, one-way ANOVA).

isoflurane on AITC-evoked currents (no detectable inhibition, $n=4)$. Thus, the S5 domain in TRPA1 appears critical for the inhibitory effects of volatile anesthetics.

Two Amino Acid Residues in S5 of TRPA1 Are Critical for the Inhibitory Effects of Anesthetics. Amino acid residues in the S5 domain are important for activation by pungent anesthetics and menthol. Notably, activation by propofol and isoflurane requires Ser876 (Ton et al., 2017), whereas both serine and Thr877 are implicated in menthol sensitivity of rodent TRPA1. We therefore examined rat TRPA1 channels bearing mutations at these positions. Notably, replacing both of these residues (S876V/T877L) abolished the
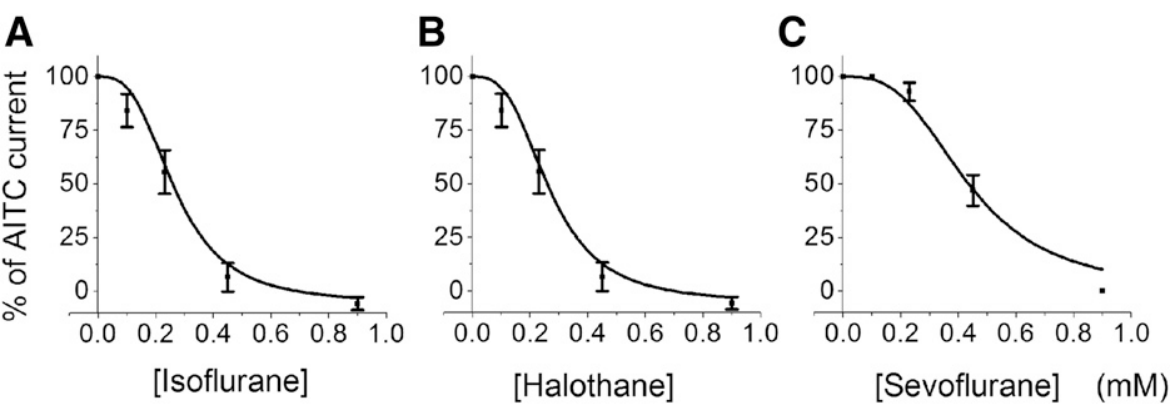

Fig. 2. Concentration-dependent inhibition of AITC-evoked currents by volatile anesthetics. Isoflurane (A), halothane (B), and sevoflurane (C) inhibit the current (measured at $-100 \mathrm{mV}$ ) evoked by AITC $(100 \mu \mathrm{M})$. The smooth lines represent fits of pooled data to a Hill equation yielding $\mathrm{IC}_{50}$ values of $0.28 \pm 0.02,0.27 \pm 0.05$, and $0.43 \pm 0.03 \mathrm{mM}$, respectively $(n=3-5$ for each concentration). 


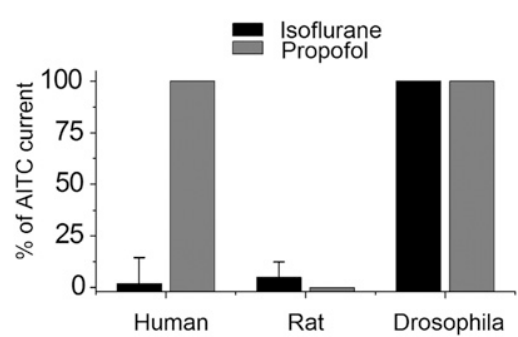

Fig. 3. Species-specific differences in the inhibitory effects of isoflurane and propofol on TRPA1 currents. Mean current measured at $-100 \mathrm{mV}$ induced by AITC $(100 \mu \mathrm{M})$ and effects of isoflurane $(0.9 \mathrm{mM})$ and propofol $(1 \mathrm{mM})$ in HEK293 cells expressing rat $(n=10)$, human $(n=5)$, and Drosophila TRPA1 $(n=4)$.

antagonist effects of isoflurane (Fig. 5B). This double mutant channel nonetheless displayed normal sensitivity to AITC. Interestingly, single mutations at either position (T877L or S876V) also mostly abolished the antagonistic effect of isoflurane. Similarly, single or double mutations of these two residues prevented the inhibitory effects of propofol, octanol, chloroform, halothane, and sevoflurane (Fig. 6). Together, these data suggest that both Thr877 and Ser876 appear essential for the inhibitory effects of anesthetics.

Ser876 and Thr877 Are Sufficient for Anesthetic Antagonism. Next we explored whether Ser876 and Thr877 are sufficient to confer anesthetic sensitivity to dTRPA1. Replacing Drosophila Leu934 with the counterpart mammalian threonine (L934T) did not convey anesthetic responsiveness (Fig. 7A). However, reintroduction of both serine and threonine (V933S/L934T) rendered the channels sensitive to the inhibitory effects of GAs ( $n=23$; Fig. 4B; Fig. 5E). Notably, isoflurane blocked AITC-evoked currents by more than $50 \%$ in these V933S/L934T receptors without producing any agonistic effects (Fig. 6A). In addition to Ser876 and Thr877, we introduced these two additional amino acids required for the binding of A-969079 and pungent anesthetics into dTRPA1 (V933S/L934T/I941L and V933S/L934T/I941L/ M1055V). Figure 7B shows that addition of these two amino acids did not alter the inhibitory effect of isoflurane compared with the V933S/L934T mutant, with a near 50\% block in all cases.

Finally, we considered the potential role for two amino acids, Met915 and Met956, in mediating inhibition. These residues are critical for activation by GAs and are proposed to directly interact with isoflurane and propofol via hydrogen/ halogen bonds and sulfur-aromatic interactions. Mutagenizing Met915 and Met956, however, did not alter the inhibitory effects (Fig. 8). Taken together, we conclude that distinct sites mediate the agonist and antagonist effects of anesthetics. Two amino acid residues (Ser876 and Thr877) are required for inhibition in mammalian TRPA1 and are sufficient to confer the inhibitory effect of anesthetics on dTRPA1.

\section{Discussion}

Several GAs are noxious, and this pharmacological effect occurs principally through the activation of the TRPA1. Here we show that in addition to selective TRPA1 activation, diverse noxious and non-noxious GAs inhibit ligand-activated TRPA1 channel activity. Half-maximal inhibition was achieved with relevant pharmacological concentrations of inhalational agents: 1 MAC of isoflurane, sevoflurane, and halothane. Propofol inhibited rodent TRPA1 with an approximate halfmaximal response of $100 \mu \mathrm{M}$, a concentration relevant to the site of injection (Doenicke et al., 1996). These inhibitory effects of GAs were seen in mammalian but not Drosophila TRPA1. One notable exception was human TRPA1, which was inhibited
A

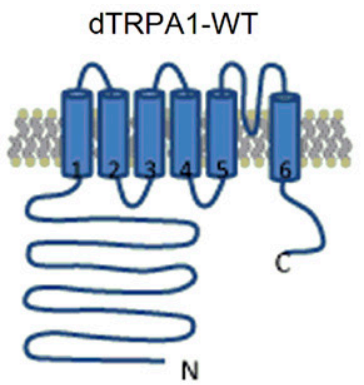

B

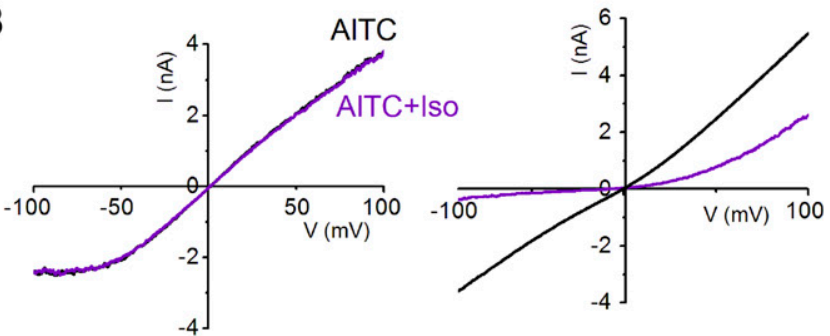

MTRPA1-WT

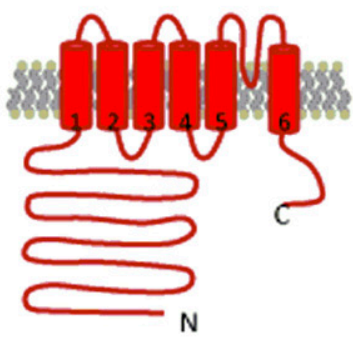

dTRPA1-mN
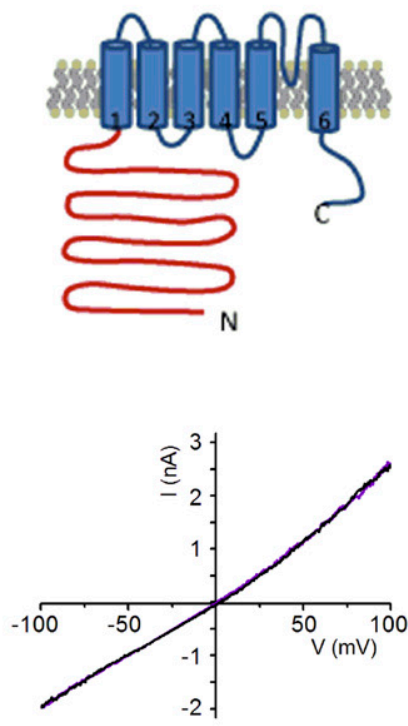

mTRPA1-dS5
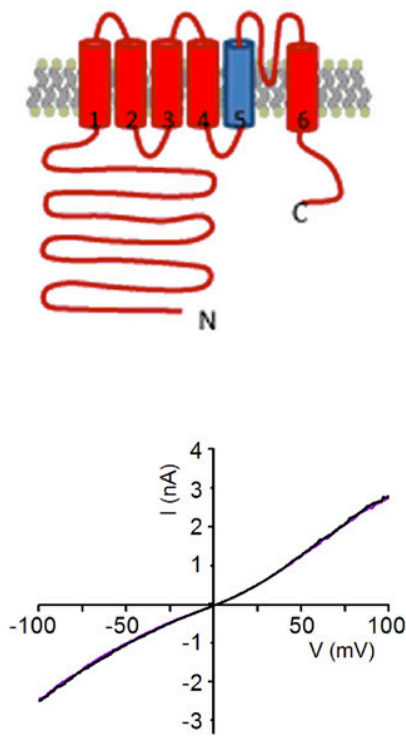

Fig. 4. Response of wild-type and chimeric mouse-Drosophila TRPA1 channels to isoflurane. (A) Schematic of receptors of wild-type dTRPA1 (dTRPA1WT), wild-type mouse TRPA1 (mTRPA1-WT), chimera made by exchanging the Drosophila $\mathrm{N}$ terminus with its mouse counterpart (dTRPA1-mN), and chimera made by introducing the Drosophila S5 domain into the mouse channel (mTRPA1-dS5). (B) Current-voltage relationship for responses to AITC and isoflurane (Iso; $0.9 \mathrm{mM})$ plus AITC $(100 \mu \mathrm{M})$ in HEK293 cells transfected with corresponding chimeric TRPA1 channels shown in A. Note that control currents were subtracted. 
A

Mouse (861-894):

Human (864-897):

Drosophila (921-954):
S5

VMLEVIFKTLIRSTGVFIFLILAFGLSFYVLI VMLEVILKTLLRSTVVFIFLLLAFGLSFYILL VMFLEILQTLIKVLMVFSILIIAFGLAFYILL

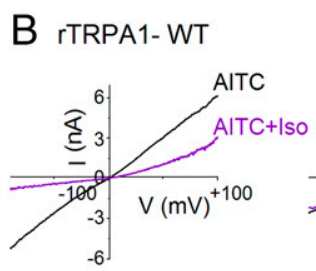

\section{S876V/T877L}

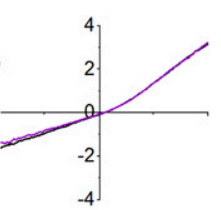

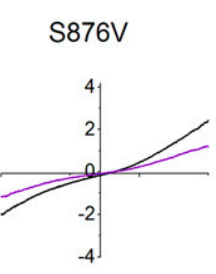

T877L

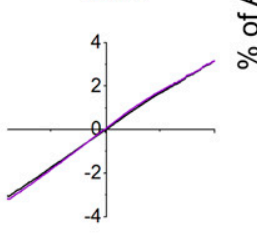

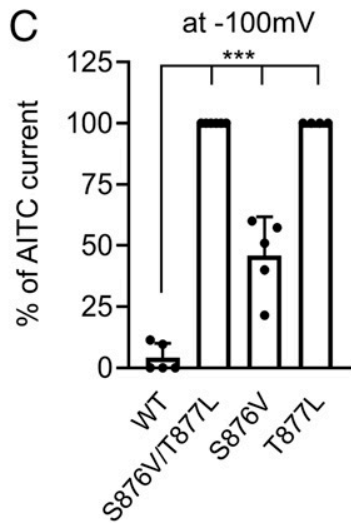

Fig. 5. Two amino acids in TRPA1 S5 domain are critical for sensing isoflurane-induced inhibition. (A) Alignment of S5 region used in chimeras from mouse, human, and Drosophila TRPA1. Amino acids in dTRPA1 divergent from the mammalian sequence are marked in yellow. (B) Representative current-voltage (I-V) traces ( -100 to $+100 \mathrm{mV}$ ) evoked by AITC (100 $\mu \mathrm{M}$, black traces) and isoflurane (Iso) plus AITC (purple traces) for wild-type rat TRPA1 (rTRPA1-WT) and mutant rat TRPA1. (C) Mean current responses of isoflurane inhibition measured at - $100 \mathrm{mV}$ in HEK293 cells expressing wild-type (WT) rat TRPA1 $(n=5)$ and its mutants [S876V/T877L $(n=6)$; S876V $(n=5)$; T877L $(n=4)]$. *** $P<0.001$, one-way ANOVA.

by inhalational GAs but not by propofol. Previous studies have shown that noxious GAs have bimodal effects on TRPA1, activating at low concentrations and inhibiting at higher concentrations (Matta et al., 2008; Eilers et al., 2010; Fischer et al., 2010). Our data extend these findings and show that volatile agents potently inhibit TRPA1 channels activated by a maximal concentration of AITC. This inhibition of AITC-activated current is consistent with an open channel block mechanism in which anesthetics inhibit by preferentially binding to the open state and therefore, the degree of inhibition is proportional to open probability. Similarly, sevoflurane inhibition of Shaker Kv channels increases with depolarization and channel activation (Lioudyno et al., 2013). If GAs inhibit TRPA1 by an open channel block mechanism, then one apparent anomaly is the voltage dependence of GAinduced block. Strong membrane depolarization activates TRPA1, yet paradoxically, this leads to relief of GAinduced inhibition (Matta et al., 2008; Ton et al., 2017). One explanation for this anomaly is that unlike $\mathrm{K}^{+}$ channels, the pore region of transient receptor potential
A

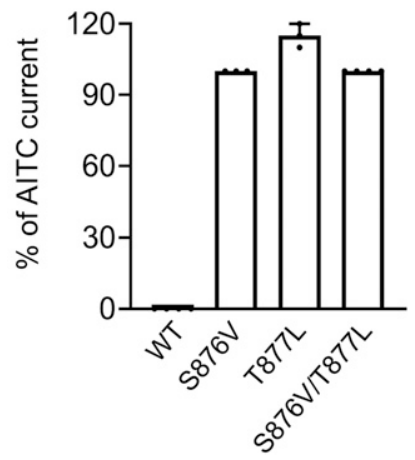

B

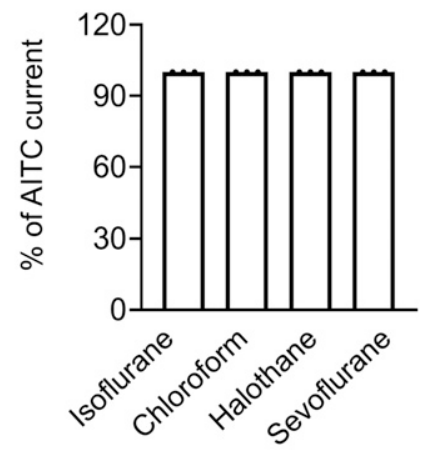

Fig. 6. Ser876 and Thr877 residues of rat TRPA1 are critical for inhibition by diverse GAs. (A) Summary of inhibition of AITC-evoked currents (measured at $-100 \mathrm{mV}$ ) by propofol $(1 \mathrm{mM})$. WT, wild type. (B) AITCevoked currents $(-100 \mathrm{mV})$ in T877L TRPA1 channels are unaffected by isoflurane $(0.9 \mathrm{mM} ; n=4)$, chloroform $(1 \mathrm{mM} ; n=4)$, halothane $(1 \mathrm{mM}$; $n=3)$, and sevoflurane ( $5 \mathrm{mM} ; n=5$ ). channels undergoes voltage-dependent structural rearrangements (Cao et al., 2013) that may prevent open channel block by GAs.

We exploited chimeric proteins and mutagenesis to identify critical sites required for these inhibitory effects. These experiments identified two critical residues in the S5 domain, Ser876 and Thr877; mutating either of them abolished inhibition. However, whether these GAs directly interact with these residues to produce inhibition is unclear. Interestingly, Ser876 and Thr877 and along with several other residues in S5 and S6 form a binding pocket for A-967079, and noxious GAs are predicted to directly act at this site to activate TRPA1 (Ton et al., 2017). However, whereas reconstituting this pocket in dTRPA1 confers activation by GAs (Ton et al., 2017), it does not confer inhibition. Furthermore, we found that mutagenesis of select residues in the A-967079 pocket (Met915 and Met956) critical for activation by GAs had no effect on inhibition. Thus, these data indicate that GA activate and inhibit TRPA1 via distinct sites and mechanisms. This conclusion is further supported by the voltage dependence of the inhibitory effect, whereby large depolarizations selectively abolished GA-induced inhibition without affecting activation (Matta et al., 2008; Ton et al., 2017) and by the distinct pharmacology of noxious and non-noxious GAs in terms of activation and inhibition of TRPA1. Of note, a recent photolabeling study also supports distinct propofol binding sites in mouse TRPA1 (Woll et al., 2017). Meta-aziproprofol labeled two residues in the S6 helix, Val951 and Glu966. Furthermore, molecular modeling suggested that Val951 is located in close proximity to the A-967079 binding pocket and the presumptive GA agonist site, whereas Glu966 is located deep in the pore near the lower gate. Interestingly, in the channel open state Glu966 is predicted to orientate to the pore and therefore may provide a substrate for GA-induced open channel block (Woll et al., 2017). Therefore, mutagenesis of Ser876 and Thr877 could relieve inhibition by disrupting these structural rearrangements that allow for propofol to penetrate the lower pore region. In this regard, it is notable that menthol has an inhibitory action in rodent but not in human TRPA1 and that these differences are attributed to variation in a single residue; Gly878 in rodents is substituted with Val875 in humans (Xiao et al., 2008). This would lead to 
A

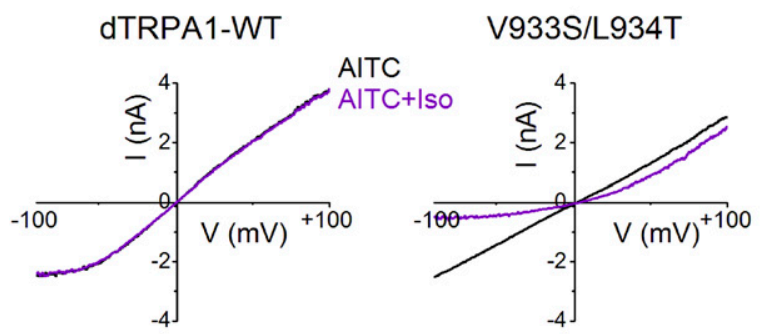

B

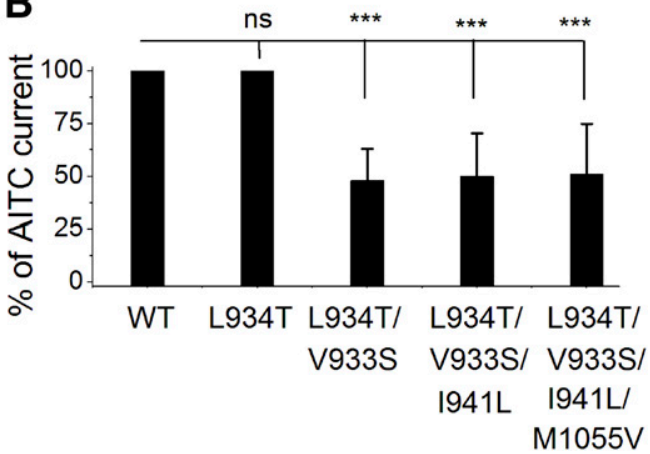

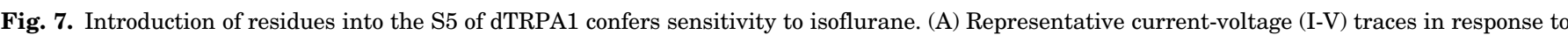

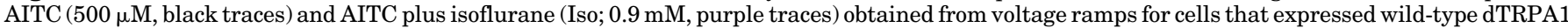

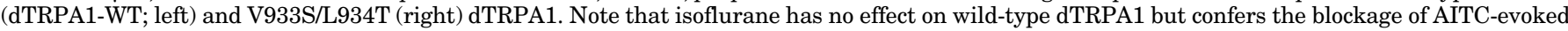

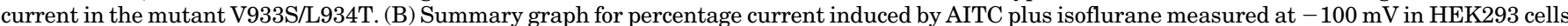

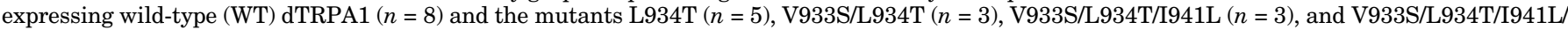
M1055V $(n=6)$. *** $P<0.001$, one-way ANOVA; ns, not significant.

an increase in side-chain mass at this position in human TRPA1 that may prevent menthol accessing an inhibitory binding pocket. Similarly, the glycine-valine substitution may explain the differential inhibitory actions of propofol that we identified in rodents and human TRPA1.

TRPA1 has an important role in pain signaling and neurogenic inflammation (Bautista et al., 2013). Therefore, inhibition of TRPA1 by pharmacological concentrations of inhalational GAs may modulate nociceptive signaling. On the other hand, it should be noted that some inhalational GAs also stimulate TRPV1 (Cornett et al., 2008), so the net effects on pain are unclear. In general, GAs are not analgesic. Propofol inhibits rodent TRPA1 at concentrations suprapharmacological for anesthesia. However, these concentrations are relevant to the free concentration of propofol at the local site of injection, estimated to be $100 \mu \mathrm{M}$ (Doenicke et al., 1996). In this regard, it is notable that propofol does not inhibit human TRPA1, and this lack of inhibition, therefore, may exacerbate propofol-induced activation of TRPA1 and the pain upon injection in humans. Indeed, selectively removing inhibition, by mutagenesis of Thr877 in rat TRPA1, enhances propofolevoked currents (Ton et al., 2017). In addition to sensory nerves, TRPA1 is expressed in vascular endothelium. Indeed, activation of TRPA1 in the vasculature may contribute to the hypotensive actions of propofol (Sinha et al., 2015). Furthermore, TRPA1 is a molecular sensor of hypoxia (Takahashi
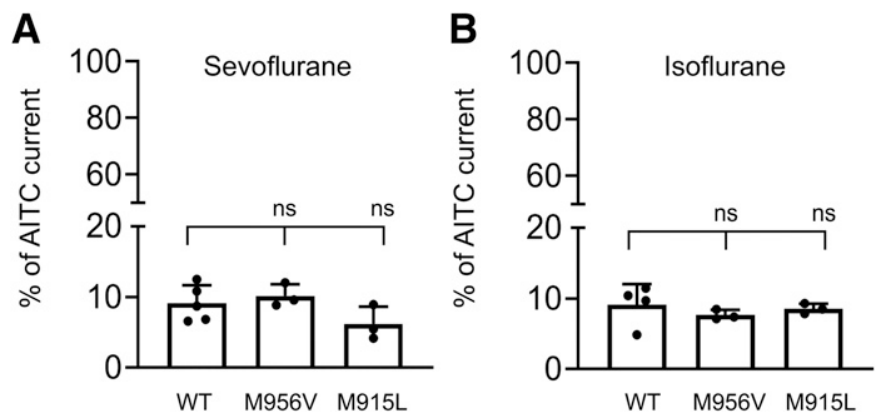

Fig. 8. Disruption of methionine residues critical for GA activation does not affect GA-induced inhibition. (A and B) Mean current induced by AITC plus sevoflurane (A) or isoflurane (B) $(0.9 \mathrm{mM})$ measured at $-100 \mathrm{mV}$ in HEK293 cells expressing wild-type (WT; $n=4)$, M956V $(n=3)$, or M915L rat TRPA1 $(n=3)$. ns, not significant. et al., 2011), and this mechanism may contribute to the vasodilation of cerebral arteries during ischemia (Pires and Earley, 2018). Thus, inhibition of TRPA1 by volatile GAs has potential important implications for the regulation of regional blood flow during ischemia. In contrast, propofol, which does not inhibit human TRPA1 and minimally blocks rodent TRPA1 at anesthetic concentrations, may exert neuroprotective properties compared with inhalational agents.

\section{Authorship Contributions}

Participated in research design: Ton, Ahern.

Conducted experiments: Ton, Phan.

Performed data analysis: Ton, Phan, Ahern.

Wrote or contributed to the writing of the manuscript: Ton, Ahern.

\section{References}

Bautista DM, Pellegrino M, and Tsunozaki M (2013) TRPA1: a gatekeeper for inflammation. Annu Rev Physiol 75:181-200.

Cao E, Liao M, Cheng Y, and Julius D (2013) TRPV1 structures in distinct conformations reveal activation mechanisms. Nature 504:113-118.

Cornett PM, Matta JA, and Ahern GP (2008) General anesthetics sensitize the capsaicin receptor transient receptor potential V1. Mol Pharmacol 74: $1261-1268$.

Doenicke AW, Roizen MF, Rau J, Kellermann W, and Babl J (1996) Reducing pain during propofol injection: the role of the solvent. Anesth Analg 82:472-474.

Eilers H, Cattaruzza F, Nassini R, Materazzi S, Andre E, Chu C, Cottrell GS, Schumacher M, Geppetti P, and Bunnett NW (2010) Pungent general anesthetics activate transient receptor potential-A1 to produce hyperalgesia and neurogenic bronchoconstriction. Anesthesiology 112:1452-1463.

Fischer MJM, Leffler A, Niedermirtl F, Kistner K, Eberhardt M, Reeh PW, and Nau C (2010) The general anesthetic propofol excites nociceptors by activating TRPV1 and TRPA1 rather than GABAA receptors. J Biol Chem 285:34781-34792.

Li F, Guo CJ, Huang C-C, Yu G, Brown SM, Xu S, and Liu Q (2015) Transient receptor potential A1 activation prolongs isoflurane induction latency and impairs respiratory function in mice. Anesthesiology 122:768-775.

Lioudyno MI, Birch AM, Tanaka BS, Sokolov Y, Goldin AL, Chandy KG, Hall JE, and Alkire MT (2013) Shaker-related potassium channels in the central medial nucleus of the thalamus are important molecular targets for arousal suppression by volatile general anesthetics. J Neurosci 33:16310-16322.

Matta JA, Cornett PM, Miyares RL, Abe K, Sahibzada N, and Ahern GP (2008) General anesthetics activate a nociceptive ion channel to enhance pain and inflammation. Proc Natl Acad Sci USA 105:8784-8789.

Mutoh T, Taki Y, and Tsubone H (2013) Desflurane but not sevoflurane augments laryngeal C-fiber inputs to nucleus tractus solitarii neurons by activating transient receptor potential-A1. Life Sci 92:821-828.

Picard P and Tramèr MR (2000) Prevention of pain on injection with propofol: a quantitative systematic review. Anesth Analg 90:963-969.

Pires PW and Earley S (2018) Neuroprotective effects of TRPA1 channels in the cerebral endothelium following ischemic stroke. eLife 7:e35316.

Satoh J and Yamakage M (2009) Desflurane induces airway contraction mainly by activating transient receptor potential A1 of sensory C-fibers. J Anesth 23:620-623.

Sinha S, Sinharoy P, Bratz IN, and Damron DS (2015) Propofol causes vasodilation in vivo via TRPA1 ion channels: role of nitric oxide and BKCa channels. PLoS One 10:e0122189. 
Takahashi N, Kuwaki T, Kiyonaka S, Numata T, Kozai D, Mizuno Y, Yamamoto S, Naito S, Knevels E, Carmeliet P, et al. (2011) TRPA1 underlies a sensing mechanism for O2. Nat Chem Biol 7:701-711.

Tan CH and Onsiong MK (1998) Pain on injection of propofol. Anaesthesia 53:468-476. TerRiet MF, DeSouza GJ, Jacobs JS, Young D, Lewis MC, Herrington C, and Gold MI (2000) Which is most pungent: isoflurane, sevoflurane or desflurane? Br J Anaesth 85:305-307.

Ton HT, Phan TX, Abramyan AM, Shi L, and Ahern GP (2017) Identification of a putative binding site critical for general anesthetic activation of TRPA1. Proc Natl Acad Sci U S A 114:3762-3767.

von Ungern-Sternberg BS, Saudan S, Petak F, Hantos Z, and Habre W (2008) Desflurane but not sevoflurane impairs airway and respiratory tissue mechanics in children with susceptible airways. Anesthesiology 108:216-224.
Woll KA, Skinner KA, Gianti E, Bhanu NV, Garcia BA, Carnevale V, Eckenhoff RG, and Gaudet R (2017) Sites contributing to TRPA1 activation by the anesthetic propofol identified by photoaffinity labeling. Biophys $J$ 113:2168-2172.

Xiao B, Dubin AE, Bursulaya B, Viswanath V, Jegla TJ, and Patapoutian A (2008) Identification of transmembrane domain 5 as a critical molecular determinant of menthol sensitivity in mammalian TRPA1 channels. $J$ Neurosci 28:9640-9651.

Address correspondence to: Gerard P. Ahern, Department of Pharmacology and Physiology, Georgetown University, Med-Dent Bldg. SW401, 3900 Reservoir Rd Washington DC 20007. E-mail: gpa3@georgetown.edu 